\title{
On the Stress Analysis of Functionally Graded Gear Wheels with Variable Thickness
}

\begin{abstract}
This paper presents the elastic solutions of the gear wheels made of functionally graded material (FGM) with variable thickness subjected to rotating loads. The material properties and wheel thickness profile are assumed to be represented by two power law distributions. Solid and hollow wheels are considered and the solutions for the stresses and displacements are given under appropriate boundary conditions. The solutions for FGM are compared with that of non-FGM, and for variable thickness and for uniform thickness. The effects of the material grading index, $\mathrm{n}$, and the geometry of the wheel on the stress and displacement are investigated. It is found that a functionally graded wheel with parabolic and hyperbolic convergent thickness profile has smaller stresses and displacements compared with that with uniform thickness. The maximum radial stress for the solid functionally graded wheel with parabolic thickness profile was not at the center, whereas for solid wheel with uniform thickness, the maximum was at the center. The results obtained suggest that an FGM gear wheel with hyperbolic convergent and parabolic concave thickness profile is more suitable compared with that of uniform thickness.
\end{abstract}

Keyword: Functionally Graded Material, Rotating Gear Wheel, Variable Thickness Disk, Elasticity 